\title{
Framing the preoperative assessment of the oncogreriatric patient. The surgeon's point of view
}

\author{
Dimitris Papagoras MD, MSc, PhD, FEBS, Maria Kanara MD, PhD, Stella Ntouvli ${ }^{1}$ MD, PhD \\ Department of General Surgery and Anesthesia ${ }^{1}$, General Hospital of Trikala, 42100 Trikala, Greece
}

Abstract: Elderly cancer patients represent a steadily rising population constituting a daily problem for any physician, who is confronted not only with medical questions but also with ethical and social dilemmas. In addition oncogeriatric patients are underrepresented or excluded from trials especially in surgery which results in a gap in practical and evidence based knowledge regarding their preoperative evaluation and treatment. Therefore the authors outline the framework a decision making process for a meaning - and success- full treatment plan has to rely on.

Keywords: oncogeriatric patients;preoperative assessment; decision making process

\section{Introduction}

As stated by the United Nations in the World Aging Report population aging has had and will have various social economic and hospital care consequences in the near future ${ }^{[1]}$.According to the relevant literature the Health Care Services worldwide have identified deficiencies in the care received by the elderly, especially the oncogeriatric patients $^{[2]}$, as a result of the absence or weakness of established pathways to which medical science end knowledge are adapted and incorporated into daily clinical praxis ${ }^{[3]}$. In particular there are four factors counteracting the substantiality, the importance and the spread of knowledge regarding the oncogeriatric patient ${ }^{[4]}$.

Firstly, the small amount of teaching hours spent on geriatric science at most medical schools all over the world; in fact, in our country, a chair of geriatric medicine does not exist in any of our seven universities.

Secondly, the lack of validated postgraduate training programs in geriatric medicine in most European countries; actually there is no recognized specialty such as geriatric medicine or geriatric surgery.

Thirdly, the shallow and low grade awareness among the majority of physicians and specially surgeons, as this subpopulation of patients older than 65 years old deserves not only a systematic multimodal perioperative approach to deal with their unique physiology and their response to surgical insult, but also requires sublime and individualized access to their cultural, social, environmental and ethical values, points of view, expectations, preferences, wishes and wills and last but not least, the gap of data and the scarce evidence based knowledge on which we could build a rational decision making progress which would also engulf a meaningful treatment plan taking into account much more the quality of life that we have to offer to these older patients rather than look solely and in an isolated way at the mortality, morbidity and long term survival rates after major elective or urgent oncologic procedures.

Copyright (C) 2017 Dimitris Papagoras MD et al (layal.mansour@live.com)

doi: $10.18686 /$ aem.v6i2.112

This is an open-access article distributed under the terms of the Creative Commons Attribution Unported License

(http://creativecommons.org/licenses/by-nc/4.0/), which permits unrestricted use, distribution, and reproduction in any medium, provided the original work is properly cited. 
Definitions - Epidemiology

Why is this issue regarding the oncogeriatric patient, by definition a cancer patient older than 65 years ${ }^{[6]}$, so important? Because it is more than common and it constitutes a problem bearing an array of challenges encountered in everyday praxis worldwide.

We will explain what is meant by the terms "common" and "important" and what these "challenges" facing the oncogeriatric patient are.

The importance of the subject on the oncogeriatric patient is related to the fact that the elderly population is steadily expanding and continuously rising as shown by demographic studies both in industrialized and developing countries, so by 2050 , it is estimated that $16 \%$ of the world's population will be aged over 65 years and that this older age group will outnumber the pediatric population of toddlers younger than 6 years - globally ${ }^{[5]}$.

Furthermore, population projection towards 2040 indicates an increase of up to $100 \%$ of age groups above 75 years. All these will have massive implications and impact on the function and organization of the health care services and systems all over the world ${ }^{[6]}$.

What are the causes of the explosive overpopulation of the elderly? They are the result of a considerable increase in the average life span; in fact, the current life expectancy at birth is unsurpassed in human history in combination with a progressive reduction in birth rates.

If we add to these epidemiologic data the observation that cancer is a disease of ageing - in fact, longevity is the most significant independent factor for developing cancer with a life time probability of almost $45 \%$ in men and $38 \%$ in women and with the highest rates of cancer and cancer death rates occurring in subjects aged 65 years or more, then it is more than obvious that doctors will be faced with caring for an increased number of elderly cancer patients ${ }^{[6]}$.

Moreover, all these demographic data indicate and underline that we are confronted with an epidemiologic time bomb for which the scientific community seems to be totally unprepared as it is demonstrated by the simple but long lasting fact that the elderly patients are still being managed on the basis of assumptions derived from studies with younger patients, assumptions that do not necessarily apply to the oncogeriatric patients ${ }^{[7]}$.

Components of ageing

But why do we have to distinguish oncogeriatric patients from their younger counterparts?

Because of their unique physiology of the ongoing process of ageing which has two important time related aspects and components: the first one refers to the simple passage of time representing the age of an individual in years and is called chronological age, and the other pertains to the age dependent deteriorative changes and functional decline of almost all organ systems of the human body, and is called biological time. Chronological and biological ages are not the same, they must not be confused with each other, and they do not go hand in hand, although functional deterioration is more frequently apparent beyond the age of 70. This is why two elderly individuals with identical chronological ages are never the same: they differ in the mental and cognitive status, the nutritional condition, the level of physical activity, the presence of comorbidities, the perception of quality of life and life expectations.i This physiologic complexity of the biological foremost process of ageing forms two extremities with two opposite phenotypes which are, on the one hand, the senescent or frail individual with cognitive impairment and numerous comorbidities who takes many drugs, has no autonomous activity, depends on the assistance of others and has limited life expectancy and, on the other, the superfit centenarian who is still capable of strenuous exercise and has an active social life ${ }^{[9]}$.

Evaluation of the oncogeriatric patient

Few if any would dispute that those in the former group, that is he frail patient, should not be considered for any aggressive surgical, interventional or chemotherapeutical approach. These patients will die much earlier from the consequences of their senescence rather than cancer and any aggressive treatment would be not only futile but also a 
representative case of overtreatment. On the contrary the latter group, that is the superfit oncogeriatric patients should not be deprived of this opportunity, because this would be a typical example of undertreatment. The main problem is that most elderly people are spread between these two extreme phenotypes of biological ageing and the dilemma is how to deal with those oncogeriatric patients in between.

Surgery may offer the highest chance of cure after cancer has been diagnosed but at the expense of complication, mortality, loss of independence and shrinking of quality of life, which can offset any oncologic advantage and benefit from surgery.

So it is no easy task at all to decide whether to operate or not especially in the case of an oncogeriatric patient who, due to his unique vulnerabilities resulting from biological ageing and the fact that his physiologic reserves are not always apparent, requires an assessment far beyond the traditional preoperative evaluation for the average adult ${ }^{[10]}$.

Frailty

To address this difficult task of the preoperative risk assessment of an older patient, it is strongly recommended that their frailty be assessed first, and then planning an appropriate cancer treatment and management be considered ${ }^{[8]}$.

Frailty is the key - term and step in the geriatric assessment representing the utmost expression of biological ageing and being the geriatric syndrome per se. It is characterized by the total inability to tolerate any - virtually and literally any - insult and can be defined by specific indicators related to the mental, cognitive, physical, nutritional condition as well as the social functionality of the individual ${ }^{[11]}$.

Frail older patients are at the highest risk for postoperative morbidity and mortality, much higher than older patients of the same chronological age but without the vulnerability of this syndrome ${ }^{[12]}$

The full-blown phenotype of frailty includes the following characteristics (Fried and Hopkins frailty index) ${ }^{[5]}$

- Unintentional weight loss

- Self reported exhaustion

- Weakness (in grip strength)

- Slow walking speed

- Low physical activity

A simple tool to estimate these characteristics is the up and go test which measures the time taken for an old seated patient to rise, walk $3 \mathrm{~m}$, turn around and return to their seat thus identifying high risk patients in ground of frailty ${ }^{[5]}$.

Sarcopenia and cachexia

Another two concepts to be aware of in the preoperative assessment of oncogeriatric patients are (a) sarcopenia, the decrease and loss of muscle mass strength and functionality.

(b) and cachexia a metabolic syndrome associated with underlying illnesses leading to muscle loss.

All these three components overlap and their presence constitutes a clear cut contraindication for major cancer surgery ${ }^{[5]}$.

\section{Individual preoperative assessment}

Two additional key elements to frailty in the pathway of the oncogeriatric assessment which have to be clarified before any definitive cancer treatment decision is made are (a) the values, preferences and expectations of the older patient ${ }^{[13]}$ and (b) the aggressiveness and invasiveness of the cancer in correlation to the corresponding type of surgery in terms of complexity and inherent life threatening complication risks ${ }^{[14]}$.

Only if these 3 matters [(frailty, preferences of the patients, and type of cancer and risk of surgery)] are approached by the physician thoroughly and honestly and with active and informed participation of the older cancer patient and his beloved ones, will the decision making process be guided to a meaningful and individualized treatment plan thus helping to avoid procedures that only prolong life without considering the components of quality of life, 
independence and dignity of the oncogeriatric patient.

\section{References}

1. The United Nations, 2014, World population aging report, viewed 25 November 2017.http://www.um.org/en/developement/ desa/population

2. Montroni I and Audisio RA, 2017, Precision surgery for older cancer patients, G Chir, vol 38(2): 59-60

3. Spiliotis J and Tepetes K, 2011, Cancer surgery in the elderly, Novabiomedical, New York

4. Masud T, Blundell A, Gordon LA, et al. 2014, European undergraduate curriculum in geriatric medicine developed using an international Delphi technique, Age and Ageing, vol. 43: 695-702

5. Schlitzkus LL, Melin AA, Johanning JM, et al. 2015, Perioperative management of elderly patients, Surg Clin N Am, vol 95:391-415

6. Soreide K and Desserud KF, 2015, Scand J Trauma Resc and Emerg Surg, vol 23:1-7

7. Rahmesh HSJ, Pope D, Gennari R, et al., 2005, Optimizing surgical management for elderly patients World J Surg Oncol, vol 3:17

8. Audisio RA, Ramesh H, Longo WE, et al. 2005, The Oncologist vol.10: 262-268

9. Korc-Grodzicki B, Downey RJ, Shahrokni A, et al. 2014, J Clin Oncol vol.32: 2647-2653

10. Tremblay D, Charlebois K, Terret C, et al., 2012, BMJ Open, vol. 2: e001483

11. Huisingh -Scheetz M and Walston J, 2017, How should older adults with cancer evaluated for frailty? J Geriatric Oncol, vol. 8: $8-15$

12. Hall DE, Arya S, Schmid KK, et al., 2017 Association of a frailty screening initiative with postoperative survival at 30,180 and 365 days, JAMA Surgery, vol. 152(3): 233-240

13. Dunn GP, 2016, Schared decision making for the elderly patient with a surgical condition, BJS, vol.103: e19-20

14. Audisio RA. Tailoring surgery to the elderly patients with cancer, 2016, BJS, vol.103:e10-11 